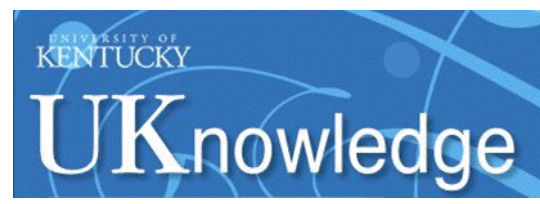

University of Kentucky

UKnowledge

11-20-1992

\title{
Modeling SiO Maser Emission from Late-Type Stars
}

Philip B. Lockett

Centre College

Moshe Elitzur

University of Kentucky, moshe@pa.uky.edu

Follow this and additional works at: https://uknowledge.uky.edu/physastron_facpub

Part of the Astrophysics and Astronomy Commons, and the Physics Commons

Right click to open a feedback form in a new tab to let us know how this document benefits you.

\section{Repository Citation}

Lockett, Philip B. and Elitzur, Moshe, "Modeling SiO Maser Emission from Late-Type Stars" (1992). Physics and Astronomy Faculty Publications. 228.

https://uknowledge.uky.edu/physastron_facpub/228

This Article is brought to you for free and open access by the Physics and Astronomy at UKnowledge. It has been accepted for inclusion in Physics and Astronomy Faculty Publications by an authorized administrator of UKnowledge. For more information, please contact UKnowledge@lsv.uky.edu. 


\section{Modeling SiO Maser Emission from Late-Type Stars}

\section{Digital Object Identifier (DOI)}

http://dx.doi.org/10.1086/171963

\section{Notes/Citation Information}

Published in The Astrophysical Journal, v. 399, no. 2, p. 704-713.

(C) 1992. The American Astronomical Society. All rights reserved.

The copyright holder has granted permission for posting the article here. 


\title{
MODELING SiO MASER EMISSION FROM LATE-TYPE STARS
}

\author{
PHILIP LOCKETT \\ Department of Physics, Centre College, Danville, KY 40422 \\ AND \\ Moshe ELITZUR \\ Department of Physics and Astronomy, University of Kentucky, Lexington, KY 40506 \\ Received 1991 August 8; accepted 1992 May 15
}

\begin{abstract}
We have performed a thorough study of both radiative and collisional pumping of the SiO masers around late-type stars, carefully considering the combined and separate actions of each type of pump in order to gauge its effectiveness. We find that collisional pumping is severely underestimated when the model calculations use a small number (less than about 18) of rotational levels in each vibrational state. We have developed a procedure that corrects this problem and gives results that are nearly independent of the number of levels utilized in the calculations. We recognize, but do not solve, an important problem that afflicts all escape probability treatments which include maser saturation effects on the level populations. Maser radiation is strongly beamed and the functional form of the beaming angle must be known to properly calculate the maser escape probability. However, the beam pattern for saturated masers in the presence of large velocity gradients has yet to be studied in the literature.

Our model is based on observations and theoretical arguments that place the SiO masers in high-density clumps rather than in the smooth stellar wind. Significantly, general conclusions can be reached which are independent of the pumping mechanism. Most importantly, the overall molecular density is restricted to lie between $\sim 10^{9}-10^{10} \mathrm{~cm}^{-3}$, regardless of the type of pumping. In addition, both collisional and radiative pumps result in the production of a maser chain within each vibrational state, as observed. There are some important differences, however, between the pumping mechanisms. All pumps based on stellar radiation become less efficient with distance from the star because of the rapid decline in pump rate. This prevents any radiative pump from being able to produce the observed maser emission over most of the observed maser region. The only feasible radiative pumps require fine tuning of physical conditions and produce inversion only over a narrow range of optical depths that depends sensitively on the size of the velocity gradient and the form of the escape probability expression. In addition, these radiative pumps have difficulty in explaining the simultaneous production of masers in the same rotational transitions of adjacent vibrational states as is observed. We find that collisional pumping produces the strongest maser emission and, in contrast to radiative pumping, generates maser radiation over the entire observed region and does not require fine tuning of the physical parameters for its operation. Furthermore, there is a significant range of overlapping column densities where collisional pumping produces maser emission in the same rotational transitions of adjacent vibrational states, as observed. Collisional pumping thus appears to be the primary pumping mechanism responsible for the $\mathrm{SiO}$ maser phenomenon.
\end{abstract}

Subject headings: masers - molecular processes - stars : late-type

\section{INTRODUCTION}

Maser emission has been detected in a number of rotational transitions in the excited vibrational states of the $\mathrm{SiO}$ molecule. Although first detected toward Orion, maser emission is most commonly observed coming from late-type stars. Unlike the $\mathrm{OH}$ and $\mathrm{H}_{2} \mathrm{O}$ masers, $\mathrm{SiO}$ maser emission is not produced in the expanding wind around these stars (Elitzur 1980). Instead, it occurs much closer to the star, inside the dust formation point before the outflow is established. Recent polarization and interferometry observations confirm that maser emission emanates from large blobs of material that have specific velocities and often well defined polarization angles (McIntosh et al. 1989). The cell lifetimes are typically a fraction of a stellar period. The relatively rapid creation and destruction of the cells makes theoretical modeling and the interpretation of observations difficult. This has led to some confusion, particularly when comparing observations of the same source at different times in the stellar cycle. However, detailed theoretical analysis of the masers offers an opportunity to learn a great deal about the physical conditions in these complex regions. This may lead to a better understanding of the processes of dust formation and mass loss from late-type stars.

An important goal of the modeling process is to determine the pumping mechanism. This is complicated for the $\mathrm{SiO}$ maser because both radiation and collisions are important in transferring molecules from the ground state into the higher vibrational states, where maser emission is observed, and both are capable of producing inversion of the rotational transitions. In order to understand the relative importance of radiation versus collisions, it is necessary to examine their separate and combined effects. Our calculations treat the separate and combined effects of collisions and radiation in order to gauge the effectiveness of each type of pump.

Two distinct types of radiative pumps have been proposed for the SiO maser. In the first scheme, formulated by Kwan \& Scoville (1974), masers in vibrational state $v$ are produced by 
first radiatively pumping molecules into the state $v+1$. Unfortunately, this calculation was hampered by an improper choice of the vibrational Einstein $A$-coefficients. One of the objectives of our study is to reexamine this pump using correct values for the radiative rate coefficients. The second type of radiative pump was proposed by Deguchi \& Iguchi (1976). This pump is potentially more efficient than that of Kwan and Scoville because it inverts the transitions in vibrational state $v$ by directly pumping molecules into that state. We performed calculations using both types of pumps and are able to offer new insights into the operation of each.

Collisional pumping was first proposed by Elitzur (1980), whose analysis showed that collisions provide a robust and efficient pump that turns on when the vibrational transitions become optically thick. The basic operation of the pump is independent of the specific values of the collision cross sections (Watson, Elitzur, \& Bieniek 1980), but numerical modeling requires their availability. Since the inception of this model, cross sections have been calculated (Bieniek \& Green 1983) that allow collisional pumping models to be analyzed in detail. We utilized these cross sections to conduct a thorough study of collisional pumping.

The SiO maser is difficult to model accurately because numerical calculations are limited to a finite number of rotational levels in each vibrational state, with serious effects on the calculated optical depths and also on the collisional pump rates. These problems have been recognized in previous models and some corrective methods have been devised (Bujarrabal \& Nguyen-Q-Rieu 1981; Langer \& Watson 1984). We have developed new methods for handling these problems that lead to results that are essentially independent of the number of levels used in the calculation. One consequence of these corrections is that the collisional pumping efficiency is significantly enhanced.

The analytical work of Elitzur (1980) and the calculations of Langer \& Watson (1984) show that the densities in the stellar wind are not sufficient to produce the observed maser strengths. Our model places the masers not in the wind, but rather in high-density clumps closer to the star. The densities in these clumps are not constrained by the stellar wind and the higher densities lead to significantly stronger maser emission. However, the density cannot be too large, since the masers become collisionally quenched. Our results constrain the molecular density to be between about $10^{9}$ and $10^{10} \mathrm{~cm}^{-3}$.

We have also recognized a fundamental problem that afflicts all escape probability treatments of saturated maser emission in late-type stars. Maser escape probability is proportional to the beaming solid angle of the maser radiation pattern. However, beaming angles of saturated masers have only been determined for the special cases of quiescent spheres and filaments and never for media with large velocity gradients. We discuss this problem in detail, but have not yet been able to formulate a proper solution.

\section{OBSERVATIONS}

Recent observations by Jewell et al. (1991) suggest that $\mathrm{SiO}$ may be the most prevalent type of circumstellar maser. Maser emission is commonly observed in the lower rotational transitions of the $v=1$ and 2 vibrational states, and less frequently in $v=3$. Very recent observations have detected the $J=5 \rightarrow 4$ transition of $v=4$ (Cernicharo, Bujarrabal, \& Santaren 1992). The ground vibrational state occasionally shows signs of maser emission, but in general its rotational levels appear to be approximately thermally populated. A maser chain within the vibrational levels exists which presently extends up to the $J=6 \rightarrow 5$ transition, which is significantly weaker than the other members of the chain (Jewell et al. 1987). The observation of similar maser chains in many different stars suggests a common inverting mechanism for the different masers.

The masers are produced relatively close to the star. Interferometry studies (Lane 1982; McIntosh et al. 1989) place the masers at $2-6 R_{*}$. Theoretical arguments also place the masers near the star inside the dust formation point. Outside the dust formation point most of the silicon is incorporated into dust leaving little to form $\mathrm{SiO}$. In addition, high temperatures are necessary to excite the $v=2,3$, and 4 masers. Finally, large column densities are required to create the optically thick vibrational transitions that are necessary for inversion (Elitzur 1980). The density in a smooth stellar wind is governed by the mass continuity equation and the observed mass loss rates are usually not large enough to create the large vibrational opacities necessary for $\mathrm{SiO}$ inversion. The $\mathrm{SiO}$ masers are thus produced not in the stellar wind but rather in a clumpy region having complicated mass motions. The existence of these maser cells is also indicated by the complicated and variable line profiles, which consist of many spikes having typical lifetimes of a fraction of a stellar period. This clumpiness has been observed by McIntosh et al. (1989), who combined VLBI and polarization measurements to create a polarization map of $\mathrm{R}$ Cas. The resulting maser structure is cellular, with each cell characterized by distinct radial velocity, linear polarization and polarization position angle.

The photon luminosities of the maser lines in the $v=1$ and 2 vibrational states are comparable in strength (Clemens \& Lane 1983), although there is a significant decrease that appears to occur at the $J=6 \rightarrow 5$ transition (Jewell et al. 1987). An exception to this pattern is the anomalously weak strength of the $v=2, J=2 \rightarrow 1$ maser, which has been observed in only a few stars (Olofsson, Rydbeck, \& Nyman 1985). Neglecting this exception, overall maser emission from $v=1$ and 2 is approximately equal. Isotropic photon luminosities for the $J=1 \rightarrow 0$ masers in $v=1$ and 2 typically range from $10^{42}$ to $10^{44} \mathrm{~s}^{-1}$, although luminosities as high as $10^{46} \mathrm{~s}^{-1}$ have been estimated for a couple of exceptionally luminous supergiants (Lane 1982). The emission from $v=3$ tends to be about 30 times weaker (Alcolea, Bujarrabal, \& Gallego 1989). The recently discovered $v=4$ maser is weaker still.

It appears that the same rotational masers in different vibrational states are produced in the same region. The most direct evidence for this is obtained using interferometry. Lane (1982) found a good correlation between the positions of the $J=$ $1 \rightarrow 0$ masers in the $v=1$ and 2 levels of the supergiant VX Sgr. More recent observations by Miyoshi (1992) have provided dramatic confirmation that these maser transitions usually occur in the same position. There is also strong indirect evidence provided by the close similarity in the line profiles of the same rotational transitions in different vibrational states (e.g., Schwartz, Waak, \& Bologna 1979; Alcolea et al. 1989). The situation is somewhat more ambiguous for the case of different maser lines in the same vibrational state since there are no interferometric measurements for this case and conclusions have been based on the comparison of line profiles. In order for such a comparison to be meaningful, the observations must be performed nearly simultaneously, which is difficult for different rotational transitions because the lines have very different frequencies. Schwartz, Zuckerman, \& Bologna (1982) have per- 
formed the only nearly simultaneous study of different masers in the same vibrational state and found the different spectra to be very similar, suggesting a common origin for these masers too. Using the same technique, Lane (1982) and Jewell et al. (1987) reached a different conclusion but their measurements were performed, for the most part, at widely different times. Jewell et al. found that the profiles of $J=6 \rightarrow 5$ and $5 \rightarrow 4$ were similar but that both were different from the profile of $J=2 \rightarrow 1$. It is probably significant that the similar profiles were observed in the span of a few days, while the $J=2 \rightarrow 1$ was observed two months later. Polarization studies may also be used to help determine the origin of the different maser lines. A recent study by McIntosh \& Predmore (1992) contains strong evidence that different masers in the same vibrational state are produced in the same region. Their observations of Mira show that all three $v=1$ masers involving $J \leq 3$ not only have excellent correlations in velocity features but also share the same polarization position angle. They conclude that the masers are produced in the same region. Although there are certainly higher order effects, it appears that all the $\mathrm{SiO}$ maser lines share a common spatial original and the same basic inversion mechanism.

Bujarrabal, Planesas, \& Romero (1987) have found a tight correlation between $\mathrm{SiO}$ and IR luminosities, although a more recent study by Allen et al. (1989) has somewhat weakened the correlation between the two variables. However, there is no correlation between the average $\mathrm{SiO}$ and IR amplitudes for individual stars (Nyman \& Olofsson 1986). Time monitoring surveys have shown that the $\mathrm{SiO}$ maser emission closely follows the IR variability, but there is typically a phase lag of about 0.1 to 0.2 between the $\mathrm{SiO}$ maximum and the optical maximum (Nyman \& Olofsson 1986; Martinez, Bujarrabal, \& Alcolea 1988).

\section{MODELING}

Theoretical models must solve the statistical rate equations for the relevant level populations, which may then be used to find the maser intensities. The equations describing the statistical equilibrium of a multilevel atom or molecule are dependent on the radiation intensity in the spectral lines connecting these levels. Thus these equations cannot be solved separately from the equations of radiative transfer for these lines. The escape probability method is commonly used to decouple the radiative transfer equations from the level population equations. The level populations are then found by solving a set of nonlinear algebraic equations (see, e.g., Elitzur 1982 for the explicit form of the equations).

Out of necessity, numerical calculations are limited to a finite number of levels. Previous studies have typically used 5-12 rotational levels in each vibrational state. This introduces two significant sources of error. The first problem arises because the high temperature of the maser region (more than $1000 \mathrm{~K}$ ) is much larger than the rotational level spacings (the rotational constant is $1.05 \mathrm{~K}$ ). Thus most of the $\mathrm{SiO}$ population will be located in higher rotational levels (particularly in the ground vibrational state) that are not included in the calculation. Unless corrections are made, all of the population will be artificially forced into only those levels retained in the calculation, resulting in a significant overestimate of the level populations. The major effect is that vibrational optical depths, a primary factor in determining when inversion occurs, will be greatly overestimated. We adopt a corrective procedure that is similar to one that was introduced by Bujarrabal \& Nguyen-
Q-Rieu (1981) and used later in the work of Alcolea et al. (1989) and Fuente et al. (1989). We account for the missing molecules by reducing the total $\mathrm{SiO}$ population by the number of molecules that would be found in the excluded rotational levels assuming them to be thermally populated. The strength of the rotational collisional cross sections makes this a reasonable approximation, especially for the ground vibrational state where most of the molecules are located. The fraction of the $\mathrm{SiO}$ population that would be found with $J \leq J_{\max }$, if the rotational levels are thermalized, is given by

$$
F=\frac{1}{Z_{R}} \sum_{J=0}^{J_{\max }}(2 J+1) \exp \left[\frac{-1.05 J(J+1)}{T}\right],
$$

where $J_{\max }$ is the largest $J$ used in the calculations and $Z_{R}$ is the rotational partition function. Since the rotational constant is much less than the temperature, $Z_{R} \simeq T / 1.05 \mathrm{~K}$. The $\mathrm{SiO}$ density used in the calculations is then found by multiplying the nominal $\mathrm{SiO}$ density by the fraction $F$. The significance of this correction may be appreciated by noting that, without it, including as many as 12 rotational levels in each vibrational state will still result in an overestimate of the populations by a full order of magnitude!

The second major effect of using a finite number of levels is that the collisional pump rate is severely underestimated. Unlike radiation, collisional coupling between levels is significant for large values of $\Delta J$, making the collisional pump rate very sensitive to the number of rotational levels included in the calculation. Langer \& Watson (1984) noted this problem and they utilized a corrective method in their calculations. We have developed a different corrective procedure that adjusts the collisional pump rate by the ratio of the total to partial pump rates. A more detailed discussion of this procedure and its effects is presented in $\S 4.1$.

An accurate treatment of the radiative transfer is difficult because of the lack of knowledge of the physical conditions present in the maser region. All models of the $\mathrm{SiO}$ maser have relied on the escape probability formalism to treat the radiative effects. Previous calculations have used the Sobolev escape probability, which assumes a large velocity gradient in a spherically symmetric medium. Most of these studies failed to produce the observed maser luminosities, primarily because they assumed that the masers were produced in the wind, where the densities are too small to create the large column densities necessary for the inversion. Alcolea et al. (1989) claim to explain the typically observed $\mathrm{SiO}$ maser photon luminosities. They apply the functional form of the escape probability in an expanding wind but implicitly remove the constraint that the wind model imposes on the density. In a smooth wind the molecular density can be written as

$$
n_{\mathrm{H}_{2}} \simeq 1.5 \times 10^{11} \frac{\dot{M}_{4}}{r_{14}^{2} v} \mathrm{~cm}^{-3},
$$

where $\dot{M}_{4}$ is the mass-loss rate (in units of $10^{-4} M_{\odot} \mathrm{yr}^{-1}$ ), $r_{14}$ is the radius (in $10^{14} \mathrm{~cm}$ ), and $v$ is the expansion velocity (in $\mathrm{km}$ $\mathrm{s}^{-1}$ ). The parameters assumed by Alcolea et al. to explain the $v=3$ masers imply mass-loss rates in excess of $10^{-5} M_{\odot} \mathrm{yr}^{-1}$. However, they detected $v=3$ emission in the stars RR Aql, $\mathrm{R}$ LMi, R Cas and R Leo, whose mass-loss rates are estimated to be, respectively, only $7 \times 10^{-7}, 7 \times 10^{-7}, 6 \times 10^{-7}$, and $3 \times 10^{-8} M_{\odot} \mathrm{yr}^{-1}$ (Lane et al. 1987). Surprisingly, the strongest $v=3$ emission comes from $\mathrm{R}$ Leo, the star with the smallest mass-loss rate. It is clear that the masers must arise in regions 
whose density is unrelated to the mass-loss rate. The use of the functional form of the wind escape probability becomes questionable in this situation. A possible justification is that these calculations describe rapidly expanding clumps so large that radiative transfer in their interior resembles that of a wind.

A more serious problem arises from the strong beaming of maser radiation. Level populations calculated using the escape probability method can properly account for the effect of saturation (Elitzur 1990). The resulting expression for the saturated maser escape probability is

$$
\beta=\alpha_{1} \frac{\Omega}{4 \pi} \exp \left[\tau\left(-l_{0}, l_{0}\right)\right],
$$

where $\Omega$ is the maser beaming angle, $\tau\left(-l_{0}, l_{0}\right)$ is the optical depth in the direction of the maximum maser gain, and $\alpha_{1}$ is a dimensionless factor of order $0.1-0.5$ depending on the geometry. The maser escape probability is thus proportional to the beaming solid angle, so the functional dependence of the beaming angle on the source properties must be known in advance. Beaming angle calculations for saturated masers are one of the most difficult tasks of maser theory because they require self-consistent solutions of the entire radiative transfer problem. This is a difficult problem even when a specific geometry is assumed and becomes yet more difficult when velocity gradients are present, since the geometry must then be determined from the nature of the assumed velocity field. To date, beaming angles of saturated masers have been determined only for spheres and filaments in which the material is quiescent (Goldreich \& Keeley 1972; Elitzur 1990; Elitzur, McKee, \& Hollenbach 1991). Beaming angles have never been determined for saturated masers subject to any kind of large velocity gradients.

This problem, afflicting level population calculations that utilize an escape probability approach and also incorporate the maser saturation effect, has not been previously recognized. Beginning with the modeling of $\mathrm{OH} 1612 \mathrm{MHz}$ masers in latetype stars by Elitzur, Goldreich, \& Scoville (1976), the effect of beaming has been ignored. This is equivalent to replacing $\Omega$, the beaming solid angle for maser radiation, in the escape probability expression with $4 \pi$, overestimating the effects of saturation on the level populations by a factor $4 \pi / \Omega$. As a result, saturation sets in very quickly over a narrow range of optical depths, an effect evident in Figure 4 of Elitzur et al. (1976). An alternative is to assume the functional form of the unsaturated beaming angle in the saturated regime, but this procedure, too, is an approximation with an unknown accuracy. This problem afflicts all models of maser pumping schemes for expanding winds that incorporate the saturation effect utilizing the escape probability formalism. It has been avoided only in calculations that determine separately the pump parameters and incorporate them later into an appropriate, detailed two-level maser model (e.g., Lucas 1980; Elitzur, Hollenbach, \& McKee 1989). Unfortunately, this procedure is not directly applicable to $\mathrm{SiO}$ because many maser levels are coupled and different maser transitions have different degrees of saturation. This important problem has not yet been solved and we are examining possible methods for addressing it. In the meantime, we have followed a common approach of inserting $\Omega / 4 \pi=1$ in the calculation of the maser escape probability. Although this procedure will overestimate the onset of saturation, the calculation of the maser strength should not be severely affected. This is because a saturated maser acts as a linear converter, converting pumping events into maser photons with an efficiency determined by the pumping mechanism, which should not be greatly affected by the neglect of beaming.

Most of our calculations have been done using a wind escape probability with a logarithmic velocity gradient $\epsilon[=(r / v)(d v / d r)]$ equal to 1 . Using $\epsilon=1$ leads to an escape probability that is the same in all directions. It is a useful approach because it yields results that may be related to those obtained for a static source by a simple scaling of the optical depths and thus can be considered a prototype model for most situations. We also performed calculations using very large velocity gradients $(\epsilon=3,5)$ to test the efficiency of the radiative pumping scheme of Deguchi \& Iguchi (1976). The efficiency of radiative pumping in this case is quite sensitive to the choice of $\epsilon$ and the results are specific to this particular choice of geometry and velocity field.

The results of previous $\mathrm{SiO}$ maser calculations have been presented in terms of a number of different quantities. These include the unsaturated maser optical depths (Deguchi \& Iguchi 1976), saturated optical depths (Alcolea et al. 1989), brightness temperature (Fuente et al. 1989), total maser luminosity (Bujarrabal \& Nguyen-Q-Rieu 1981), and photon luminosity per radius (Kwan \& Scoville 1974; Langer \& Watson 1984). Our results are presented in terms of either the brightness temperature or photon luminosity per radius. The total isotropic luminosity can be found by integration of the luminosity per radius over the size of the maser region. The absolute value for these quantities is uncertain due to the saturated beaming effects discussed earlier. However, our values are in good agreement with those obtained by Alcolea et al. (1989) and Fuente et al. (1989), when performed using the same parameters and assumptions. We note that our calculation of the saturated maser emission has made use of the results developed by Elitzur (1990). Equations (2.4), (3.9), and (3.17) of that paper can be combined to give the following expression for the saturated maser intensity

$$
I=\frac{\alpha_{1}}{\alpha} \epsilon_{v} l_{0} e^{\tau},
$$

where $l_{0}$ is the size of the maser region, $\epsilon_{v}=\phi(v) h v A_{i j} N_{i} / 4 \pi$ is the emission coefficient, $\tau$ is the overall optical depth of the entire maser (including saturation effects), and $\alpha_{1} / \alpha$ is a geometric factor of order unity, which for simplicity we set equal to 1 .

\section{PUMPING MECHANISMS}

The primary factor that affects all pumping mechanisms is the monotonic decrease of vibrational decay rates with $J$ when the vib-rot transitions become optically thick (Kwan \& Scoville 1974). This decrease in the loss rate with $J$ results in inversion even for pump rates that are "flat," i.e., independent of $J$. This fundamental inverting mechanism is always present and, as a result, $\mathrm{SiO}$ maser emission is easy to produce with either collisional or radiative pumping. We performed extensive investigations of both types of pumps as described below.

\subsection{Collisional Pumping}

A major obstacle to the development of collisional pumping models is the lack of accurate cross sections. Fortunately, in the case of the $\mathrm{SiO}$ maser, a significant simplification can be made using the infinite order sudden (IOS) approximation, 
which is valid when the rotation energy spacings are small compared to the collisional energies. This should be a good approximation since the $\mathrm{SiO}$ rotational level spacings are small compared with the relevant gas temperature (more than $1000 \mathrm{~K})$. Watson et al. (1980) used this approximation to show that the collisional pump rate from the ground state into level $(v, j)$ is equal to the overall $0 \rightarrow v$ collisional excitation rate and is thus independent of $J$. The existence of a $J$-independent (flat) pump rate is a result of the IOS approximation and does not rely on the exact values of the individual state to state cross sections. Watson et al. (1980) and Elitzur (1980) showed that a robust collisional pumping mechanism arises once the vibrational decays become optically thick. These studies were not able to produce detailed results due to the lack of specific cross sectional data. The availability of the Bieniek \& Green (1983) cross sections allows a more complete study of collisional pumping to be performed. A significant result of the Bieniek and Green calculations is that the rotational collision cross sections are significantly larger than had been previously assumed, resulting in collisional quenching of the masers at molecular densities above $\sim 10^{10} \mathrm{~cm}^{-3}$. The IOS approximation was used by Bieniek and Green to provide a table of collisional rate coefficients for transitions ending in $J=0$. The rate coefficients for arbitrary final $J$ are then found from

$$
k_{v_{i}, j_{i} \rightarrow v_{f}, j_{f}}^{\mathrm{IOS}}=g_{j_{f}} \sum_{j=\left|j_{i}-j_{f}\right|}^{j_{i}+j_{f}}\left(\begin{array}{ccc}
j_{i} & j_{f} & j \\
0 & 0 & 0
\end{array}\right)^{2} g_{j} k_{v_{i}, j \rightarrow v_{f}, 0}^{\mathrm{IOS}}
$$

where $g_{j}=2 j+1$. It is important to emphasize that this complicated sum involving $3-j$ symbols must be used to calculate the remaining rate coefficients, if a flat pump rate is to be achieved. Langer \& Watson (1984) introduced a simpler expression (eq. (B9) of their paper) to generate the rate coefficients. However, this approximation does not generate flat pump rates but instead produces pump rates that decrease with $J$, making it more difficult to creat inversion, particularly for the $J=1 \rightarrow 0$ maser. (When $n$ rotational levels are retained in each vibrational state, the Langer and Watson pump rate into $J=0$ is larger than that into $J=n-1$ by a factor of $\sim 2 n-1$.)

Bieniek and Green only provided vibrational rates for the $v=1 \rightarrow 0$ and $v=2 \rightarrow 1$ transitions. The remaining rates must be estimated. We have used the following values for the other vibrational transitions $\left(k_{v v^{\prime}}\right)$ in most of our calculations:

$$
\begin{array}{cccc}
k_{20}=0.1 k_{21} & k_{30}=0.01 k_{21} & k_{31}=0.1 k_{21} & k_{32}=k_{21} \\
k_{40}=0.001 k_{21} & k_{41}=0.01 k_{21} & k_{42}=0.1 k_{21} & k_{43}=k_{21}
\end{array}
$$

These values are comparable to, but in general smaller than, those used by Langer \& Watson (1984). Our choice was guided by the review of molecular vibronic collisions by Sidis (1990). As noted by Langer and Watson, we find these cross sections to have little effect on the results, particularly for the $v=1$ and 2 masers.

Unlike radiation, collisions connect rotational states that differ greatly in $J$. Thus the calculated collisional pump rate into a specific rotational level is severely underestimated if only a few rotational levels are included in each vibrational state. This can be corrected in a simple manner. In the IOS approximation, the total vibrational collision rate $\left(k_{v_{i} \rightarrow v_{f}}\right)$ is given by a sum over all rotational levels of $g_{j} k_{v_{i}, j \rightarrow v_{f}, 0}^{\mathrm{IOS}}$. A correction factor can be obtained by finding the ratio of the true total collision rate (provided by Bieniek and Green) to the approximate total collision rate found from summation over a finite number of levels. This ratio is

$$
R=\frac{k_{v_{i} \rightarrow v_{f}}}{\sum_{j=0}^{J_{\max }} g_{j} k_{v_{i}, j \rightarrow v_{f}, 0}^{\mathrm{IOS}}} .
$$

An accurate corrective procedure is to multiply the individual vib-rot cross sections by $R$. Calculations show that $R$ decreases from $\sim 7$ to $\sim 1.5$ as the number of rotational levels retained in each vibrational state increases from 7 to 17 . Using seven rotational levels and no correction may result in an underestimation of the maser emission by up to three orders of magnitude! However, our correction method produces results that are approximately the same, no matter how many levels are used in the calculation.

Calculations show that collisional pumping leads to strong masers and also results in a maser chain occurring in each vibrational state. Figure 1 illustrates the emission of the masers in $v=1$. As expected, the relative strengths of the maser lines are controlled by the optical depths of the vibrational transitions. When these become thick, there is a range of column density where many transitions are inverted. As the column density increases, rotational collisions across the maser levels destroy the inversion. This occurs first for the high $J$ masers because they have the largest vibrational opacities and thus suffer more radiative trapping resulting in smaller net radiative decay rates. When the calculated maser intensities reach the observed strengths, the maser chain extends up to about $J=5$, in agreement with observations. The relative intensities of the masers in the chain are in approximate agreement with those found by Langer \& Watson (1984), except our model produces stronger emission in $J=1 \rightarrow 0$. The $J=2 \rightarrow 1$ and $J=1 \rightarrow 0$ masers are inverted over a wider range of optical depth and have the largest total emission. The total maser luminosity in these lines can be seen to be of the order of the typically observed value of $\sim 10^{43} \mathrm{~s}^{-1}$.

The relative strengths of maser lines in different vibrational states follow a similar pattern of dependence on optical depth. The higher vibrational state masers become strong at larger

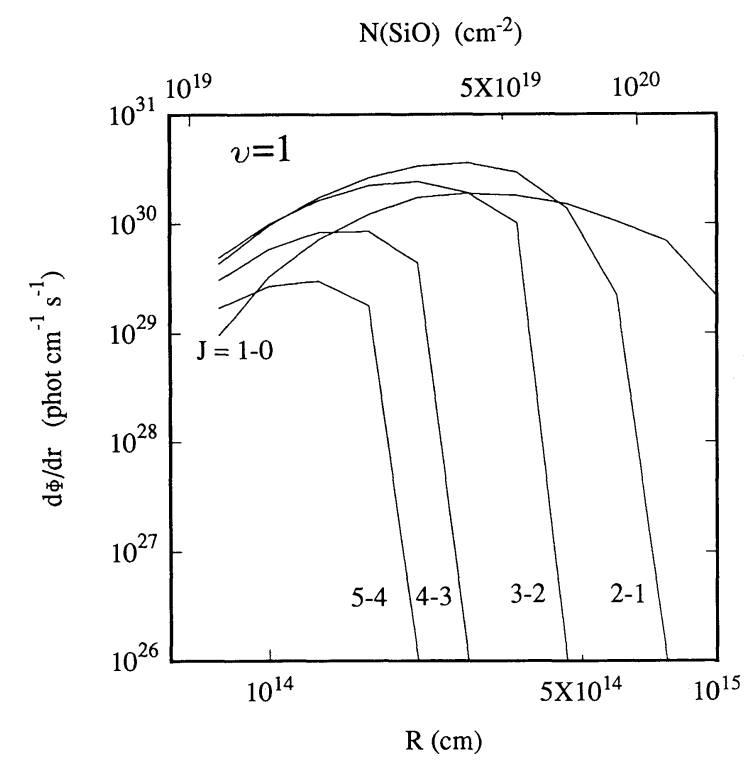

FIG. 1.-Photon luminosity per radius for the masers in $v=1$ as a function of distance from the star for a collisional pump with $T_{g}=1500 \mathrm{~K}, n_{\mathrm{H}_{2}}=$ $3 \times 10^{9} \mathrm{~cm}^{-3}$, and $X_{\text {SiO }}=5 \times 10^{-5}$. 


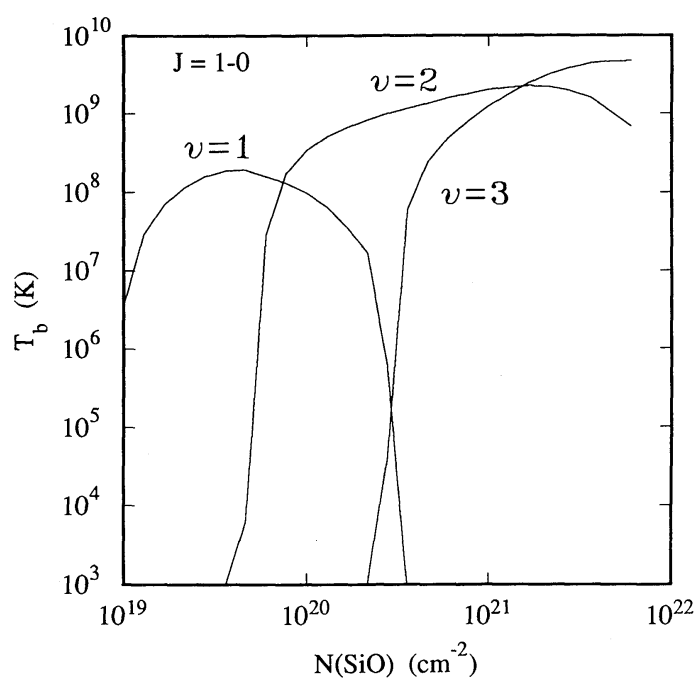

Fig. 2.-Brightness temperature for the $J=1 \rightarrow 0$ masers of $v=1,2,3$ as a function of $\mathrm{SiO}$ column density for a collisional pump with $T_{g}=1500 \mathrm{~K}$, $n_{\mathrm{H}_{2}}=10^{9} \mathrm{~cm}^{-3}$, and $X_{\mathrm{SiO}}=5 \times 10^{-5}$ (see discussion following eq. [3.4] for the method used to calculate brightness temperature).

values of column density as may be seen in Figure 2, where the emission of the $J=1 \rightarrow 0$ masers in $v=1,2$, and 3 is shown. This behavior, also seen in the work of Alcolea et al. (1989) and Fuente et al. (1989), is a direct consequence of the vibrational transitions becoming optically thick. It is significant that there is a region of overlap where the $v=1$ and 2 masers are both strong, in agreement with observations. $v=3$ masers, on the other hand, require $\mathrm{SiO}$ column densities in excess of $10^{20}$ $\mathrm{cm}^{-2}$. These column densities are more rarely achieved and thus detected $v=3$ maser emission will be rarer and weaker than that from $v=1$ or 2 . The newly discovered $v=4$ maser in VY CMa requires even larger column densities to produce maser emission. These large column densities will be found only in special sources such as the luminous supergiant VY CMa. It is also not surprising that the first maser to be discovered in $v=4$ is in a higher rotational transition, $(J=5 \rightarrow 4)$. A characteristic of all pumping mechanisms is that the higher rotational masers tend to be stronger when the masers in a given vibrational state first turn on.

Pumping mechanisms must also be able to explain the time variability of the masers. A significant factor affecting collisional pumping is the increase in the collisional pump rate with temperature. Figure 3 displays the maser emission in the $v=1, J=1 \rightarrow 0$ transition as a function of kinetic temperature. There is a significant increase (by about two orders of magnitude!) in the maser emission as the temperature increases from 1000 to $1600 \mathrm{~K}$. Temperature changes of this magnitude are predicted in shock calculations of the maser producing region (e.g., Bowen 1989).

Elitzur et al. (1989) discovered that the numerical results of their collisional pumping model of the water maser could be accurately represented by a scaling parameter $\xi$, basically the maser emission measure. The corresponding variable for $\mathrm{SiO}$ masers is defined here by

$$
\xi \equiv\left(\frac{n_{\mathrm{H}_{2}}}{10^{9} \mathrm{~cm}^{-3}}\right)^{2}\left(\frac{X_{\mathrm{SiO}}}{10^{-5}}\right)\left(\frac{R}{10^{14} \mathrm{~cm}}\right)\left(\frac{10^{5} \mathrm{~cm} \mathrm{~s}^{-1}}{v}\right),
$$

where $X_{\mathrm{SiO}}=n_{\mathrm{SiO}} / n_{\mathrm{H}_{2}}, R$ is the radius and $v$ is the expansion velocity. The scaling units have been chosen to be representative of the conditions existing in the $\mathrm{SiO}$ maser region. Elitzur

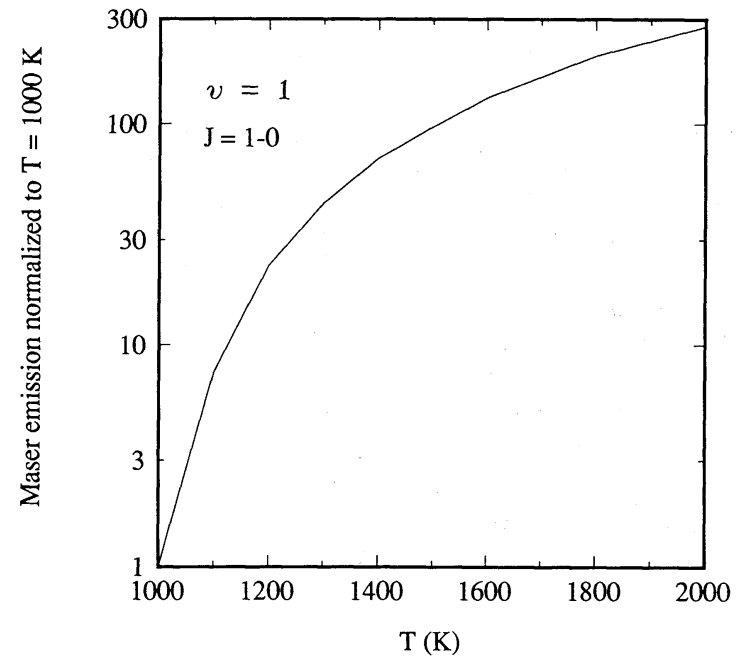

Fig. 3.-Emission for the $v=1, J=1 \rightarrow 0$ maser as a function of gas temperature for a collisional pump with $n_{\mathrm{H}_{2}}=2 \times 10^{9} \mathrm{~cm}^{-3}$ and $X_{\mathrm{SiO}}=$ $5 \times 10^{-5}$. Results are normalized to $T=1000 \mathrm{~K}$.

et al. showed that saturated maser emission at a given temperature is determined uniquely by $\xi$, when the relevant optical depths are large. Figure 4 shows the relationship between $\mathrm{SiO}$ maser emission (in the $v=1, J=1 \rightarrow 0$ transition) and $\xi$ for a range of densities. It can be seen that saturated maser emission is described reasonably accurately by $\xi$ for densities extending up to about $5 \times 10^{9} \mathrm{~cm}^{-3}$ (the flat portions of the different curves are nearly the same). Above this density rotational collisions significantly reduce the maser strength. The addition of an external radiation field complicates the dependence of the maser emission on $\xi$. The major effect is that smaller densities result in peak emission occurring at smaller values of $\xi$. Although this effect is noticeable for masers inside $\sim 4 R_{*}$, it becomes unimportant at larger distances.

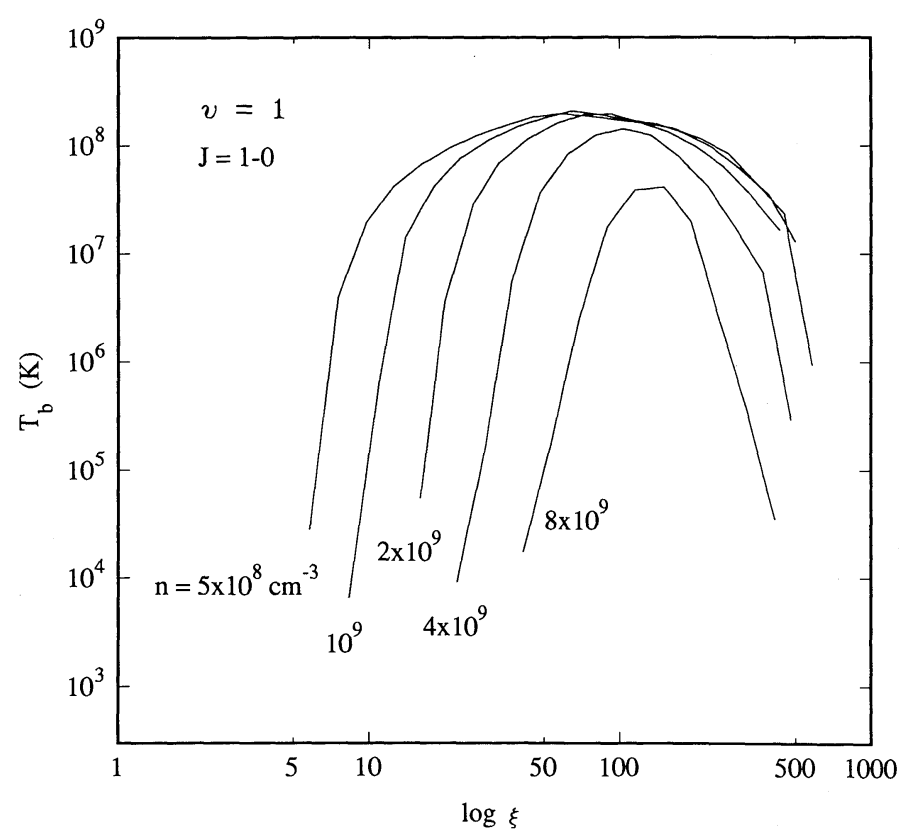

Fig. 4.-Brightness temperature for the $J=1 \rightarrow 0$ maser of $v=1$ as a function of the dimensionless parameter $\xi$ for a range of densities at $T_{g}=1500 \mathrm{~K}$. (see eq. [4.3] for the definition of $\xi$ ). 


\subsection{Radiative Pumping}

Two fundamentally different radiative pumping mechanisms have been proposed. The first is due to Kwan \& Scoville (1974, hereafter KS). This is an indirect pump that inverts the rotational levels of vibrational state $v$ by cycling molecules through the higher vibrational state $v+1$. It is essentially a $\Delta v=2$ pump. The second, due to Deguchi \& Iguchi (1976, hereafter $\mathrm{DI})$, is a $\Delta v=1$ pump that relies on the existence of a very large radial acceleration $(\epsilon>1)$. Since the pump rates for $\Delta v=1$ transitions are much larger than those for $\Delta v=2$, the DI pumping mechanism could be more efficient than the KS mechanism. We have reanalyzed these pumps and are able to offer new insights into their operation.

The original KS radiative pump assumed that the vibrational transitions $v=0-1$ and $v=0-2$ are both optically thick, while $v=1-2$ is thin. This results in the production of $J=1 \rightarrow 0, J=2 \rightarrow 1$ and $J=3 \rightarrow 2$ masers in $v=1$. However, no other maser emission is produced in either the $v=1$ or 2 vibrational states. In order to invert rotational transitions of vibrational state $v$, this model requires that the transition $v+1 \rightarrow v$ be thin and $v \rightarrow v-1$ thick, simultaneously. This precludes maser emission within adjacent vibrational states from occurring in the same region, in disagreement with observations. In addition, there is no inversion for rotational transitions beyond $J=3 \rightarrow 2$. However, this analysis was based on inadequate assumptions concerning the relative optical depths of the important vibrational transitions.

We have reanalyzed the problem using proper assignments for the relevant opacities and found that in the maser region the transitions $v=0-1$ and $v=1-2$ are both optically thick, while $v=0-2$ is thin. This combination of optically thin pumping $(v=0 \rightarrow 2)$ and optically thick decay $(v=2 \rightarrow 1)$ directly inverts the lower rotational transitions of $v=2$. The inversion is then transferred to $v=1$ by cascade from $v=2$. This leads to significant differences from the original $\mathrm{KS}$ model. Most importantly, it allows maser emission from $v=1$ and 2 to be produced in the same region. Furthermore, the maser chain does not stop at $J=3$ but continues to higher rotational levels.

We have developed approximate analytical expressions for the level populations that accurately reproduce the results of numerical calculations. Our analysis neglects collisions and assumes that the rotational levels of the ground state are thermally populated. The populations per sublevel, $n_{(v, J)}$, for $v=2$ are found by equating the radiative excitation rate to the radiative decay rate. The inversion occurs because the excitation rate from $v=0 \rightarrow 2$ is optically thin, while the radiative decay rate from $v=2 \rightarrow 1$ is optically thick. This leads to the following expression for the populations:

$$
n_{(2, J)} \simeq P \frac{n_{(1,0)}}{2}\left[K_{21}(2 J+1) A_{20} n_{(0,0)} J_{20}+2 J_{21}\right],
$$

where $J_{v v^{\prime}}$ is the average intensity in the vibrational pump line $\left(v-v^{\prime}\right)$ of the stellar radiation field,

$$
K_{21}=\frac{c^{2} R}{8 \pi v_{21}^{2} \Delta v_{21}}
$$

and

$$
P=\frac{c^{2}}{2 h v_{21}^{3}}
$$

Equation (4.4) illustrates the increase with $J$ of the populations of the rotational sublevels of $v=2$. The populations for $v=1$ are found in a similar manner. In this case, the relevant transitions $(v=2-1$ and $v=1-0)$ are both optically thick, and the rates into and out of the levels have the same dependence on $J$. The inversion of the rotational levels of $v=2$ is then transferred directly to those of $v=1$ and the level populations are given by a particularly simple expression:

$$
n_{(1, J)} \simeq \sqrt{\frac{n_{(0,0)}}{2}\left[n_{(2, J+1)}+n_{(2, J-1)}\right]} .
$$

The KS pump relies on small optical depths for the transitions that absorb the pump photons and this property prevents the pump rates from becoming large. We find that the peak maser emission of the KS radiative pump is significantly less than that of a collisional pump acting alone. In order to achieve a more efficient radiative pump, DI proposed a model where $\Delta v=1$ pumping could directly cause inversion. This model is based on the anisotropic trapping of stellar radiation in a region having a very large velocity gradient $(\epsilon>1)$. When this occurs, the optical depth is smallest in the radial direction, producing an asymmetry between the absorption of stellar IR photons and the escape of the emitted radiation. The radiative pump rates are proportional to $\beta^{*}$, the escape probability averaged over the solid angle subtended by the star, which is closely approximated by the probability for escape in the radial direction. The decay rates, on the other hand, are proportional to $\beta$, the escape probability averaged over all angles. The level populations will thus be proportional to the ratio $\beta^{*} / \beta$ and because the optical depths increase with $J$, population inversion can occur if there exists a region where $\beta^{*} / \beta$ is a monotonically increasing function of its argument. The variation of $\beta^{*} / \beta$ as a function of optical depth for various values of the velocity gradient is displayed in figure 1 of DI, which makes it clear that this mechanism will produce inversion only if $\epsilon>1$ and then only over a narrow range of optical depth that depends sensitively on the actual value of $\epsilon$.

It is important to note that the crucial factor necessary for the operation of the DI mechanism is the presence of different opacities in the radial and tangential directions. Large velocity gradients are not necessary to achieve this effect. The value of $\epsilon$ merely serves to define the effective geometry of the region. A disk-shaped clump whose axis is aligned with the direction to the star would also be subject to the DI mechanism. In this case, inversion is controlled by the ratio of disk radius to thickness and occurs only for specific values of this ratio.

The results of our numerical calculations using two vibrational levels agree well with the DI analysis. We performed additional calculations utilizing five vibrational states and values of $\epsilon=3$ and 5, which correspond to the velocity gradients that have been used in previous models (DI; Bujarrabal \& Nguyen-Q-Rieu 1981; Alcolea et al. 1989). Figures $5 a$ and $5 b$ illustrate the results of our calculations of the maser emission for $\epsilon=1$ and 5. The two panels differ only in the choice of the stellar radiation dilution factor, corresponding, respectively, to inner $\left(R \sim 2 R_{*}\right)$ and outer $\left(R \sim 5 R_{*}\right)$ masers. It can be seen that both radiative pumps are significantly weaker than the collisional pump at distances which are likely to delineate the outer edge of the maser region. We emphasize that the KS mechanism operates in each of these cases and naturally occurs when the vib-rot transitions become optically thick. The DI mechanism, however, is only activated when $\epsilon>1$ and then only a narrow range of optical depth that depends on the 

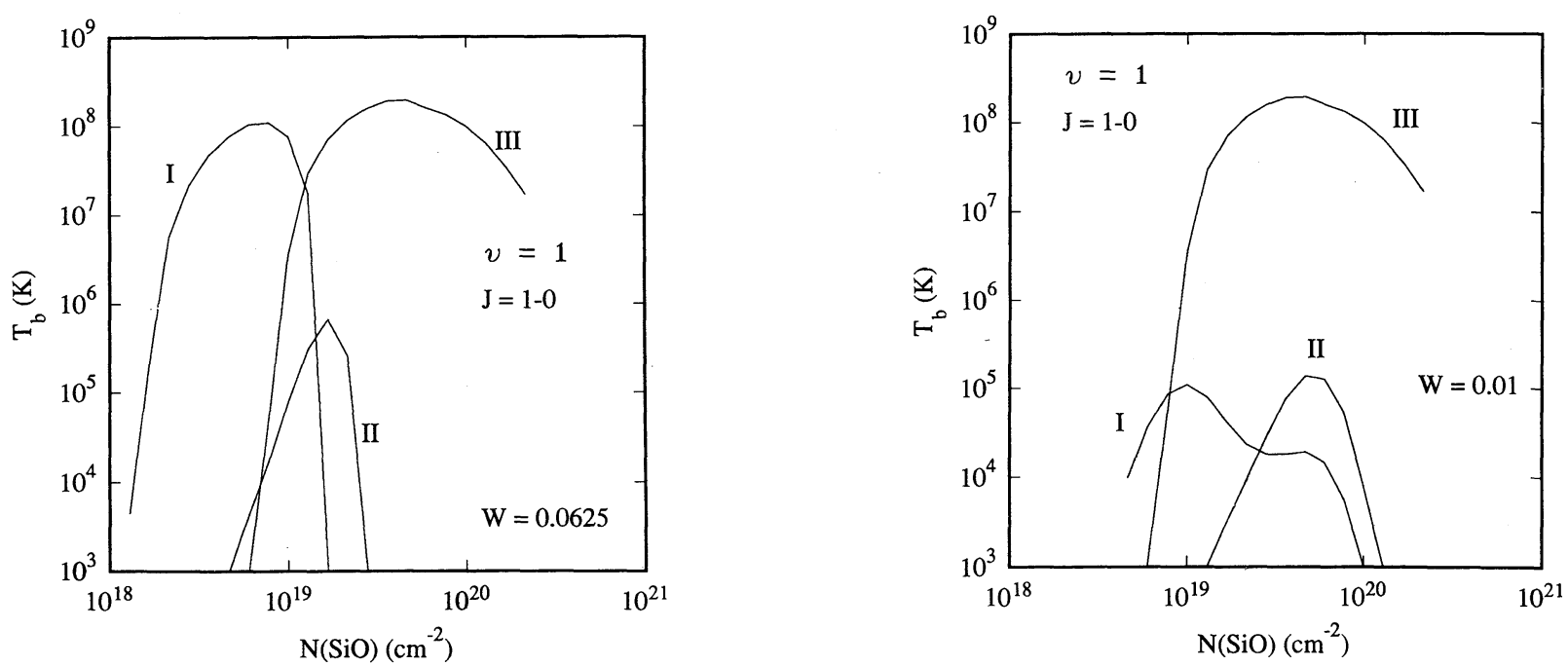

FIG. 5. - (a) Brightness temperature for the $v=1, J=1 \rightarrow 0$ maser as a function of SiO column density with $T_{g}=1500 \mathrm{~K}, n_{\mathrm{H}_{2}}=2 \times 10^{9} \mathrm{~cm}^{-3}, X_{\mathrm{SiO}}=5 \times 10^{-5}$. The different plots are for:

I: radiative pump with $\epsilon=5$ (dominated by the DI mechanism),

II: radiative pump with $\epsilon=1$ (only KS mechanism activated),

III: Collisional pump, $\epsilon=1$.

Plots I and II assume a radiation temperature $=2500 \mathrm{~K}$ with a dilution factor $=0.0625$, corresponding to $R \sim 2 R_{*}$. (b) Same as Fig. $5 a$ except that the dilution factor is 0.01 , corresponding to $R \sim 5 R_{*}$. Note that the bimodal appearance of plot I illustrates the separate effects of the DI and KS mechanisms.

choice of $\epsilon$. We find that the peak maser emission for $\epsilon=1$ or 3 are essentially the same, while $\epsilon=5$ does lead to a significant increase in emission. We also find that using $\epsilon>1$ causes the peak maser emission to occur at a smaller value of the column density. A similar effect may be found by comparing the results of Alcolea et al. (1989) (who used $\epsilon=3$ ) to those of Fuente et al. (1989) (who used $\epsilon=1$ ).

\subsection{Combined Collisional and Radiative Pumping}

The combined effects of radiative and collisional pumping are complicated and depend on a number of variables; e.g., molecular density, and kinetic and stellar temperatures. In general, we find that collisional pumping alone leads to the strongest masers. The addition of external radiation tends to decrease the maser emission, an effect that was already noted by Langer \& Watson (1984) and Alcolea et al. (1989). A similar antagonistic relationship between radiative and collisional pumping was found in modeling water masers (Cooke \& Elitzur 1985; Neufeld \& Melnick 1991). Figure 6 shows the separate and combined effects of collisions and radiation on the emission of the $v=1, J=1 \rightarrow 0$ maser. The maser emission produced when both pumps are present tends to be intermediate between the emission produced when they act separately.

\section{DISCUSSION}

The effect that dominates $\mathrm{SiO}$ maser emission is the decrease of vibrational decay rates with $J$ when the vib-rot transitions become optically thick. The inversion patterns shown in Figures 1 and 2 for collisional pumping are qualitatively the same for all pumps and some conclusions can be reached which are pump independent.

First, rotational collisions result in maser quenching at total molecular densities of $\simeq 10^{10} \mathrm{~cm}^{-3}$. This quenching density is consistent with the results of other models (e.g., Alcolea et al. 1989 ) that have used the Bieniek and Green cross sections. This upper limit to the density makes it more difficult to recreate the large optical depths required for strong maser emission. Earlier models (e.g., Elitzur 1980) had assumed smaller rotational col- lision rates which resulted in larger quenching densities. Although the Bieniek and Green results are not exact, they believe that the total rotational rates are unlikely to be in error by more than $10 \%$.

Second, the maser chains observed within the vibrational states are a natural result of both collisional and radiative pumping. The relative maser strengths of the transitions within a given vibrational state can be made to agree fairly well with observations for reasonable values of the physical parameters. However, a persistent problem is that the $J=1 \rightarrow 0$ maser is underestimated in strength; it exceeds the $J=2 \rightarrow 1$ maser only when that maser is about to turn off. This result is also

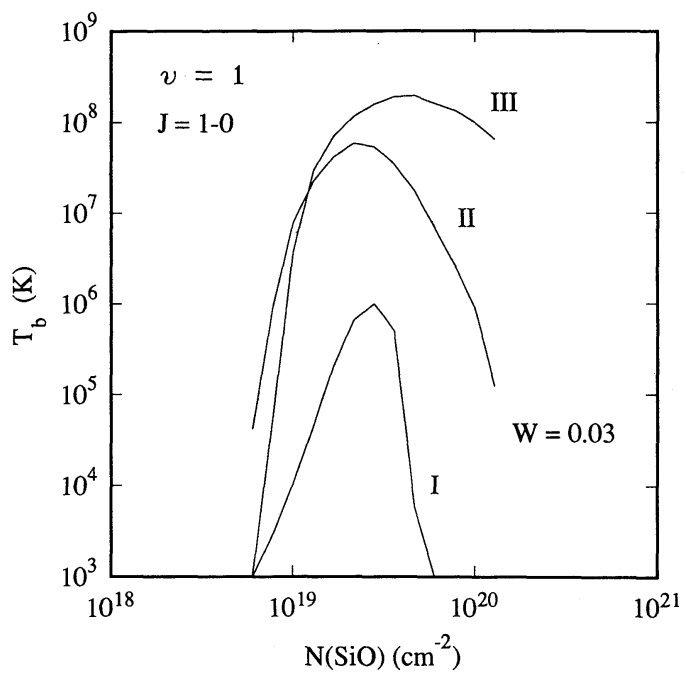

FIG. 6.-Brightness temperature for the $v=1, J=1 \rightarrow 0$ maser as a function of SiO column density with $T_{g}=1500 \mathrm{~K}, n_{\mathrm{H}_{2}}=2 \times 10^{9} \mathrm{~cm}^{-3}, X_{\mathrm{SiO}}=$ $5 \times 10^{-5}$. The different plots are for:

I: Radiative pumping only,

II: Combined radiative and collisional pumping,

III: Collisional pumping only.

The radiative pump assumes a radiation temperature $=2500 \mathrm{~K}$ and a dilution factor $=0.03$ corresponding to $R \sim 3 R_{*}$. 
found in all previous studies that have examined the higher rotational masers (e.g., Bujarrabal \& Nguyen-Q-Rieu 1981; Langer \& Watson 1984). Part of the problem may be due to the anomalously weak $v=2, J=2 \rightarrow 1$ maser. This anomaly cannot be modeled without introducing some asymmetry in the transition rates for the involved levels. The most promising proposal is that line overlap is involved (Olofsson et al. 1985). By adjusting the rates to weaken this maser line, the $J=1 \rightarrow 0$ maser strength is also enhanced.

Third, the masers in higher vibrational states require larger column densities for their production. The $v=3$ and 4 masers will be rarer and weaker since they require much larger column densities for their production. The column densities required for peak maser emission in the different vibrational states are essentially the same for collisional pumping and for radiative pumping with $\epsilon=1$. Radiative pumping with $\epsilon>1$ requires somewhat smaller column densities. A lower limit for the molecular density may be estimated from the $\mathrm{SiO}$ column density, maser size and $\mathrm{SiO}$ abundance. Our results (and those of Fuente et al. 1989) predict that the $v=1$ and 2 masers both become intense for $\mathrm{SiO}$ column densities between $10^{19}$ and $10^{20} \mathrm{~cm}^{-2}$. Assuming that the masers are $\sim 10^{14} \mathrm{~cm}$ in size and that most of the silicon goes into $\mathrm{SiO}$ gives a lower bound of $\sim 10^{9} \mathrm{~cm}^{-3}$ for the molecular density. The overall molecular density is thus restricted to lie between $\sim 10^{9}-10^{10} \mathrm{~cm}^{-3}$ regardless of the pumping mechanism.

Although many important properties of collisional and radiative pumps are similar, it is important to distinguish their separate effects and determine which is the dominant factor in producing the $\mathrm{SiO}$ maser emission. Previous studies have concentrated on the combined action of radiation and collisions and have not completely distinguished their separate effects. KS performed the only study, that we are aware of, that compared a purely radiative pump to a combined radiative and collisional pump. They found that collisions significantly increase maser emission over that achieved by radiation alone. Out of necessity, they had to rely on guesses for the collision cross sections and the values they used for the vibrational collision rates are much too large. Furthermore, they restricted vib-rot collisions to $\Delta J=0, \pm 1$, when much larger values of $\Delta J$ are actually allowed. However, these two assumptions tend to cancel in the calculation of the total collisional pump rate and the total KS pump rates are of the same order of magnitude as those found by Bieniek and Green. Furthermore, the expression assumed by $\mathrm{KS}$ produces a nearly flat pump rate. In spite of the inadequacies in the $\mathrm{KS}$ radiative and collisional rates, their finding that collisions provide the more effective pump is accurate.

The relative contributions of collisional and radiative pumping are also a function of the number of rotational levels kept in the calculation. The discussion in $\S 4.1$ emphasizes that the collisional pump rate is severely underestimated if a small number of levels is used with no correction factor for the collision rate. The importance of this effect can be seen by comparing our work with that of Fuente et al. (1989). They present results obtained from a model that uses only five rotational levels in each vibrational state. We reproduce their results accurately when we use the same level structure and no correction to the collision rates. However, when the number of rotational levels in each vibrational state is increased to 18 , the maser strengths are significantly increased and the column density where peak maser emission occurs is also changed. The results using 18 levels are essentially identical with an without our corrective procedure, and increasing further the number of levels has no significant effect on the results. Finally we repeated the calculations using the original Fuente et al. five-level structure in conjunction with the correction to the collision rates. The results are essentially the same as those found using 18 levels! We amphasize that even though our corrective method gives results which are nearly independent of the number of levels used, it is still important to use as many rotational levels as is feasible in the calculation. If the collisional correction factor is not used, we recommend that a minimum of $\sim 18$ rotational levels be retained in each vibrational state.

An important obstacle facing all stellar radiative pumps is the rapid decrease in pump photon flux with distance from the star. Because of this effect, none of our radiative pumps was able to produce the observed emission for masers located at $\sim 5-6 R_{*}$. Furthermore, we rule out altogether the KS radiative pump $(\epsilon=1)$ as a viable mechanism because it is not able to produce the required emission for masers located at any distance from the star. The DI pump $(\epsilon>1)$ is capable of producing the required emission for masers located at $\sim 2-4 R_{*}$, but it requires accurate fine tuning of the physical conditions. In particular, it relies on a rapidly expanding medium centered on a source of pump photons. This could possibly work if the masers were produced in the wind, but it is difficult to see how it could work if the masers arise in clumps.

Direct radiative pumps also have a problem in producing strong $J=1 \rightarrow 0$ masers in both $v=1$ and 2 at the same position in space. The interferometry measurements of Miyoshi (1992) clearly show the precise alignment of these masers in the majority of observations. Figure 2 illustrates (for a collisional pump) the large amount of overlap in column density where the $J=1 \rightarrow 0$ masers in $v=1$ and 2 are both strong. Figure 7 is the corresponding result for a direct radiative pump. There is essentially no region of overlap where these masers are both strong. The same conclusion can be reached upon examination of Figure 9 of the paper by Alcolea et al. (1989), who utilized a direct radiative pump with $\epsilon=3$.

In contrast with radiative pumping, collisional pumping relies much less on the specific details of the conditions in the

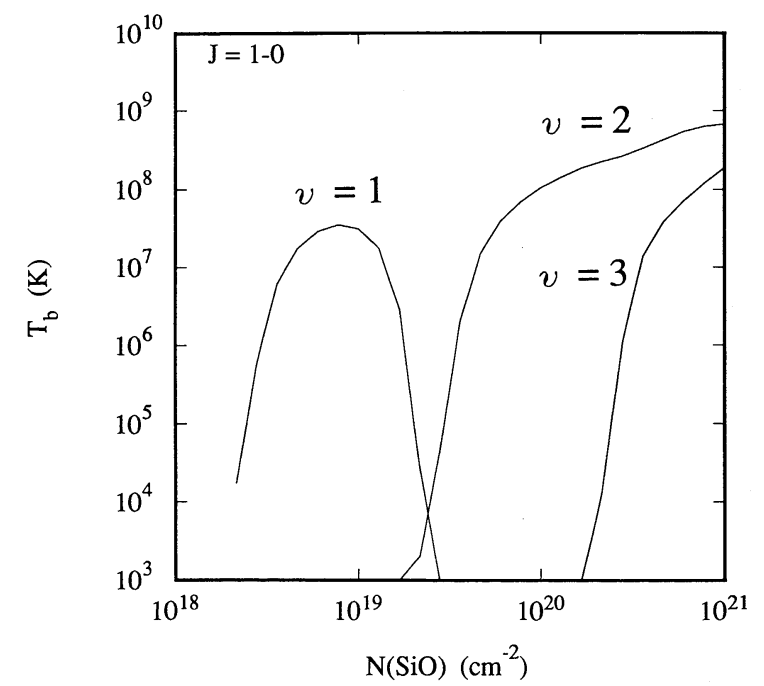

FIG. 7.-Brightness temperature for the $J=1 \rightarrow 0$ masers of $v=1,2,3$ as a function of $\mathrm{SiO}$ column density for a direct radiative pump with $\epsilon=5$, radiation temperature $=2500 \mathrm{~K}$, and dilution factor $=0.03$, corresponding to $R \sim 3 R_{*}$. (Compare to the corresponding case of collisional pumping shown in Fig. 2.) 
maser region. All that is required are molecular densities from $\sim 10^{9}$ to $10^{10} \mathrm{~cm}^{-3}$ and kinetic temperatures greater than about $1200 \mathrm{~K}$. In addition to the effects of temperature, the variation in $\mathrm{SiO}$ maser intensity could also be due to changes in density. Although this would affect all pumping mechanisms, the intensity of a collisionally pumped maser varies quadratically with density, when the $\mathrm{SiO}$ abundance is constant. This could lead to the large observed changes in the maser emission.

Since propagating shocks are likely to be important in determining local conditions in the atmosphere (cf. Bowen 1989), more accurate modeling of their effect on $\mathrm{SiO}$ masers will require the detailed results of shock models. Additional support for collisional pumping and for the importance of shocks was provided recently by Heske (1989), who observed a close correlation between $\mathrm{SiO}$ maser and $\mathrm{H} \alpha$ emission in a study of late-type stars. She proposes a collisional pumping model where shock waves create the high densities and temperatures necessary to produce the observed emission.
In conclusion, collisional pumping is a robust inversion mechanism that operates under a wide range of physical conditions and, in all likelihood, is the primary pump mechanism responsible for $\mathrm{SiO}$ masers. Although this mechanism appears to provide a satisfactory explanation for the ubiquitous presence of $\mathrm{SiO}$ masers around late-type stars, detailed comparisons with observations are difficult due to the great complexity of the extended atmospheres. Radiative pumping requires fine tuning of specific physical conditions and thus is less likely to be the dominant mechanism for these masers, whose occurrence is widespread among all types of red giants and supergiants. While radiative pumping is unlikely to be the primary cause of the masers, it cannot be completely ruled out and may occasionally be the primary pumping mechanism for certain observed features.

We thank V. Bujarrabal for very useful discussions. This work was supported by NSF grant AST-9016810 and by a grant from the Kentucky EPSCoR Program.

\section{REFERENCES}

Alcolea, J., Bujarrabal, V., \& Gallego, J. D. 1989, A\&A, 211, 187

Allen, D., Hall, P., Norris, R., Troup, E., Wark, R., \& Wright, A. E. 1989, MNRAS, 236, 363

Bieniek, R. J., \& Green, S. 1983, ApJ, 265, L29

Bowen, G. 1989, in IAU Colloq. 106, Evolution of Peculiar Red Giant Stars, ed. H. Johnson \& B. Zuckerman (Cambridge: Cambridge Univ. Press), 269

Bujarrabal, V., \& Nguyen-Q-Rieu. 1981, A\&A, 102, 65

Bujarrabal, V., Planesas, P., \& del Romero, A. 1987, A\&A, 175, 164

Cernicharo, J., Bujarrabal, V., \& Santaren, J. L. 1992, in Astrophysical Masers, ed. A. Clegg \& G. Nedoluha (NY: Springer), in press

Clemens, D. P., \& Lane, A. P. 1983, ApJ, 266, L117

Cooke, B., \& Elitzur, M. 1985, ApJ, 295, 175

Deguchi, S., \& Iguchi, T. 1976, PASJ, 28, 307 (DI)

Elitzur, M. 1980, 240, 553 1982, Rev. Mod. Phys., 54, 1225 1990, ApJ, 363, 638

Elitzur, M., Goldreich, P., \& Scoville, N. 1976, ApJ, 205, 384

Elitzur, M., Hollenbach, D. J., \& McKee, C. F. 1989, ApJ, 346, 983

Elitzur, M., McKee, C. F., \& Hollenbach, D. J. 1991, ApJ, 367, 333

Fuente, A., Martin-Pintado, J., Alcolea, J., \& Barcia, A. 1989, A\&A, 223, 321

Goldreich, P., \& Keeley, D. A. 1972, ApJ, 174, 517

Heske, A. 1989, A\&A, 208, 77

Jewell, P. R., Dickinson, D. F., Snuder, L. E., \& Clemens, D. P. 1987, ApJ, 323, 749
Jewell, P., Snyder, L. E., Walmsley, C. M., Wilson, T., \& Gensheimer, P. 1991, A\&A, 242, 211

Kwan, J., \& Scoville, N. 1974, ApJ, 194, L97 (KS)

Lane, A. P. 1982, Ph.D. thesis, Univ. Massachusetts

Lane, A. P., Johnston, K. J., Bowers, P. F., Spencer, J. H., \& Diamond, P. J. 1987, ApJ, 323, 756

Langer, S., \& Watson, W. 1984, ApJ, 284, 751

Lucas, R. 1980, A\&A, 84, 36

Martinez, A., Bujarrabal, V., \& Alcolea, J. 1988, A\&AS, 74, 273

McIntosh, G., \& Predmore, C. R. 1992, in preparation

McIntosh, G., Predmore, C. R., Moran, J. M., Greenhill, L. J., Rogers, A. E. E., \& Barvainus, R. 1989, ApJ, 337, 934

Miyoshi, M. 1992, in Astrophysical Masers, ed. A. Clegg \& G. Nedoluha (NY: Springer), in press

Neufeld, D. A., \& Melnick, G. J. 1991, ApJ, 368, 215

Nyman, L.-A., \& Olofsson, H. 1986, A\&A, 158, 67

Olofsson, H., Rydbeck, O. E. H., \& Nyman, L.-A. 1985, A\&A, 150, 169

Schwartz, P. R. Waak, J. A., \& Bologna, J. M. 1979, AJ, 84, 1349

Schwartz, P. R., Zuckerman, B., \& Bologna, J. M. 1982, ApJ, 256, L55

Sidis, V. 1990, in Advances in Atomic, Molecular, and Optical Physics, Vol. 26, ed. D. R. Bates \& B. Bederson (San Diego: Academic Press), 161

Watson, W. D., Elitzur, M., \& Bieniek, R. J. 1980, ApJ, 240, 547 